\title{
History of EPR Studies from the H.M. Swartz Laboratories: Part 2-EPR Biodosimetry
}

\author{
Harold M. Swartz ${ }^{1}$ iD
}

Published online: 9 January 2022

(c) The Author(s), under exclusive licence to Springer-Verlag GmbH Austria, part of Springer Nature 2022

\section{Overview of Research on Biodosimetry in Laboratories Directed by Harold (Hal) Swartz}

Four of my first six papers (from 1964 to 1968) involved biodosimetry, which was an inadvertent byproduct of the main goals for my first full-time research position. I was trained by the army as a Nuclear Medical Officer and assigned to the Walter Reed Army Institute of Research (WRAIR). My assignment at WRAIR was in a program focused on developing drugs to protect against the effects of ionizing radiation, with an emphasis of using EPR to follow the impact of the drugs on radiation-induced free radicals. While I carried out the complex preparations for accomplishing my principal research task, I completed several studies looking at radiation-induced free radicals in biological systems. My first publication mentioned the potential use of EPR techniques to measure radiation dose utilizing cold temperature stabilized hydroxyl radicals ${ }^{1}$. It was clear, however, that techniques requiring very cold temperatures would not be suitable for use in in vivo retrospective biodosimetry.

As detailed below, these initial background studies carried out at my WRAIR laboratory demonstrated the potential of EPR for biodosimetry. Most notable and widely cited was the 1968 reference suggesting the use of teeth, bone, and nails for biodosimetry in settings where victims were potentially at acute risk $^{6}$. Then, my research returned to the WRAIR laboratory's primary focus on radioprotective drugs and went in other directions, as covered in a companion history of work involving free radicals ${ }^{573}$.

\footnotetext{
Editors' Note: Because there is considerable overlap in the references in the three histories of EPR studies from H.M. Swartz laboratories, references with Swartz as an author use a common numbering system. These numbers are from HMS' curriculum vita and are noted by superscript numbers in this text. The full references are published only in the accompanying editorial, Part 2 of the Special Issues of AMR on the Occasion of Harold M. Swartz's $85^{\text {th }}$ Birthday [1]. References included below with this history are those that do not include Swartz as a coauthor.
}

Harold M. Swartz

Harold.Swartz@Dartmouth.edu

1 Department of Radiology, Geisel School of Medicine, Dartmouth College, Hanover, NH, USA 
I lost interest in EPR biodosimetry as a line of my research at that time, because it seemed clear to me that the technique, while interesting and perhaps useful for small radiation accidents or epidemiological studies of historic exposures, had no clear pathway for using it for the purpose of most interest to me: in large-scale settings involving recent life-threatening exposures. This conclusion was based on the impracticality of using the only EPR method then available, i.e., in vitro EPR spectroscopy on isolated teeth. Although I was intrigued with the potential for dosimetry based on clippings of nails, I chose not to pursue the latter because of the priorities for other parts of my research agenda.

Many years later, after we had developed the capability of making measurements in vivo with EPR, our instrumental innovations provided an opportunity to return to biodosimetry, with a very clear emphasis on refining EPR techniques to be used to aid in medical triage of victims after a large event involving ionizing radiation. This led a very large series of accomplishments and publications (detailed below), including a new emphasis for our group on how to fit biodosimetry into the response to a major unplanned radiation event. The attractiveness of our approaches was indicated by the very substantial funding (reaching $\$ 10$ million/year for several years) that we received for this purpose from the US Departments of Defense (DOD) and Health and Human Services (HHS) and indirectly from Homeland Security (DHS).

\section{Details of Our Research in EPR Biodosimetry}

\subsection{Initial Studies in Model Systems}

While the potential for long-lived radiation-induced EPR signals to occur in isolated and dried bones and teeth had been previously shown [2-4], the field of EPR biodosimetry might reasonably be said to have been initiated in 1965 with the publication in Radiation Research: "Long-lived electron spin resonances in rats irradiated at room temperature" ${ }^{2}$. Samples measured in vitro from rats that had been irradiated in vivo and then sacrificed showed detectable radiation-induced EPR signals in the relatively dry tissues of animals, i.e., bones, teeth, nails, and hair. ${ }^{1}$ In rat teeth, using the then state-of-the-art EPR, I estimated we could resolve doses as low as one gray (Gy).

The overall parameters for the practical use of EPR biodosimetry were laid out in the abstract published in Health Physics in $1968^{\prime \prime 6}$

Several tissues, especially hard tissues, showed persistent electron spin resonances following in vivo or in vitro irradiations. The resonances had a linear relation to dose. Dose measurements were made in teeth at less than 100 rads ( $1 \mathrm{~Gy}$ ) of $\mathrm{Co}^{60}$ radiation. The method appears to be applicable for dosimetry of accidental irradiations, especially X- or gamma-ray exposures.

\footnotetext{
${ }^{1}$ Blood appeared to be a potentially valid fifth type of tissue ${ }^{3}$, but it was shown later to be an artifact related to the generation of nitric oxide.
} 
In this study, titled "In vivo dosimetry by electron spin resonance spectroscopy", the spectra were measured at $-196{ }^{\circ} \mathrm{C}$. While most data were from rats irradiated in vivo and then sacrificed, it also was shown that readily detectable EPR signals were seen in vitro at room temperature in human bone from an accidental irradiation (estimated dose $\sim 100 \mathrm{~Gy}$ to a subject who later died) and from a human fingernail clipping irradiated to $40 \mathrm{~Gy}$. Radiation-induced signals that were stable at room temperature also were found in rat hair.

The fundamental findings underpinning much of EPR dosimetry were first outlined in this publication ${ }^{6}$, i.e., that

1. Teeth can be a robust biodosimeter for clinically relevant doses (i.e., at least down to $1 \mathrm{~Gy})$.

2. The EPR signal in teeth has long-term stability (first claimed to be stable for at least several weeks and later shown to be for thousands of years).

3. The relationship between magnitude of the EPR signal and dose is linear in bone, teeth, and nails, i.e., a linear dose-response appeared to be present in the tissues studied.

4. The dose-added method for calibration of each specimen can be used. (Retrospectively, this method was found to have been previously suggested by Duchesne and colleagues [5] in an attempt to do archeological dating of bones by EPR.)

5. Dosimetry based on nails is feasible.

6. The EPR signals in bone are associated with the mineralized portion of the bone. (It is logical for this to be true in teeth too, but I did not directly confirm it in this study.)

7. The dose-response for the radiation-induced EPR signals in teeth is more robust than that in bones. The signals in bone seem too weak for retrospective dosimetry at doses of interest for triage, but might be useful for reconstruction of accidents in which there was a large but non-whole-body dose that resulted in a fatality or amputation, so that a bone sample in the affected area could reasonably be obtained.

8. While an EPR signal was seen in the hair of the irradiated rats, hair was later understood to be an unlikely practical dosimeter for triage because of the presence of the EPR signal in melanin, typically present in the pigment of most hair. (The initial studies were done in rats with white hair, so the radiation-induced signal was observable.)

As mentioned above, my studies in biodosimetry using EPR took a 20-year hiatus at this point. We became involved again in radiation dosimetry as I became aware of a line of research based on EPR measurements in exfoliated teeth to retrospectively measure radiation dose in subjects many years after their exposure to radiation. There were two principal lines of this research, one from survivors of the atomic bombs in Japan and the other from people living in areas with a very high-level radioactive contamination of the environment. Contaminants were from negligent processing of nuclear materials or accidental release of large amounts of radiation into the environment (principally in the Union of Soviet Socialist Republics). 
I also became aware of a parallel area of research in which EPR signals in the teeth of animals and other calcified tissues were used as an alternative to carbon dating (based on knowing the annual background radiation rate and total accumulation of EPR signals from background radiation in the sites such as a cave where the ancient, calcified material was located).

While, in principle, these studies could have flowed logically from the methods, results, and implications reported in our dosimetry paper in $1968^{6}$, at least some of the studies appear to represent independent (re)discovery of the phenomena we had previously reported. Nevertheless, when I began to attend meetings where the uses of EPR dosimetry were reported, the participants generously adopted me as the founding father of their field, even if it was not always true for many of the active investigators.

\subsection{Transition to EPR Measurements Made In Vivo and Securing Major Funding for Them}

When I moved to Dartmouth, I was able to get funding for my third EPR Center. ${ }^{2}$ This funding for the EPR Center for the Study of Viable Systems at Dartmouth provided the opportunity to recruit a small but very talented core of engineers with additional expertise. Then, with additional funding available from an NIH Program Project grant (originally awarded to me at my EPR Center at University of Illinois at Urbana-Champaign and transferred to Dartmouth to develop EPR oximetry), I had the extraordinary opportunity to provide long-term support for outstanding engineers, especially Ted Walczak and Piotr Lesniewski from Krakow. These in turn led to the EPR Center's being able to attract a series of excellent 'postdoctorate fellows' from around the world to spend a few years working in the EPR Center. With these extraordinary individuals and the participation of many key collaborators, we were able to develop an innovative and unique set of EPR instrumentation to perform in vivo EPR, with a focus on its eventual use in human subjects. (See elsewhere ${ }^{573}$ for instrument development history in my laboratories.)

With our initial development of in vivo $\mathrm{EPR}^{151}$, I resumed experimental studies in biodosimetry, recognizing that it might be possible to measure human teeth in vivo. This would enable us to overcome the logistical and socially unacceptable problem of needing to have a removed tooth to make EPR measurements in vitro. Now, we had the potential to use EPR measurements for prompt evaluation of radiation exposure for an acute event without pulling teeth.

In a kind of prelude to the true biodosimetry described below, we carried out our first in vivo measurements of radiation-induced EPR signals in biopolymers ${ }^{250}$. Taking advantage of the facts that many biopolymers are sterilized by high doses of radiation and that many polymers when irradiated have persistent EPR signals, we showed that we could follow the integrity of the biopolymers in vivo in mice, as the breakdown of the polymer structure led to the disappearance of the EPR signals,

\footnotetext{
${ }^{2}$ For a fuller history of the EPR Centers I founded, see the history of free radical work in my laboratories ${ }^{573}$
} 
which occurs when water penetrated to the sites of the radiation-induced unpaired electrons. Interestingly, currently, some groups are using the EPR signals in irradiated polymers or glass in objects carried by individuals as a type of indirect biodosimeter (especially the glass in cell phones!).

The potential for carrying out EPR biodosimetry on teeth within the mouth had previously been recognized by Motoji Ikeya [6]. However, his original attempts to make the measurements in the human mouth, in which he used higher frequency $(9 \mathrm{GHz})$ spectrometers, were not very productive due to the low sensitivity and unacceptable perturbations from placing an X-Band resonator on the teeth.

At the lower frequency of L-Band (used in our laboratory), non-resonant losses became quite manageable, and a much larger volume of enamel could be interrogated. After a long and sometimes painful series of studies and attempts to obtain funding for the full development of in vivo dosimetry, we succeeded during the last $20+$ years in establishing the full feasibility of in vivo tooth dosimetry. Doing so required overcoming both technical and conceptual barriers. In some ways, the technical barriers were the easiest (but not easy!), but first I will describe the conceptual barriers.

The conceptual barriers, paradoxically, arose in great part because of the successes and long prior history of using in vitro EPR tooth dosimetry. By the time we began to carry out in vivo EPR tooth dosimetry, the capabilities of in vitro tooth dosimetry had become widely established as very effective tools for retrospective studies of radiation dispersal into the environment. They became, and remain, perhaps the most effective approach to looking at long-term effects of sub-lethal levels of ionizing radiation dispersed into the environment. Providing a historical quantitative assessment of radiation exposure, they became an indispensable tool for the critically important field of determining the long-term consequences, especially carcinogenesis, of exposure to moderate doses of ionizing radiation.

These successes in using the in vitro tooth dosimetry technique were widely known and appreciated in the radiation-response community. Therefore, in our initial attempts to secure interest and, especially funding, for in vivo EPR dosimetry, we kept running into barriers arising for people's knowledge that EPR tooth dosimetry required isolated teeth and the extraction of the enamel, and could not grasp that this did not need to be true. (As a somewhat complementary and complicating aside, others made the logical suggestion that it might be feasible to simply biopsy the tooth and then measure a very small sample of enamel at $35 \mathrm{GHz}$, but this never gained much traction despite its validity.)

At first, I did not understand why, after making a presentation about in vivo tooth dosimetry, I would get inappropriate questions about their concerns for extracting the teeth, which, of course, were not relevant to measuring teeth in vivo. There was also a puzzling lack of enthusiasm for our approach, which seemed to be based mostly if not entirely on such misunderstandings.

When I finally understood the problem, I realized that straightforward explanations were only a small part of the solution and that persistent repetition of the facts was needed, so that people truly understood that we were not making ex vivo measurements. 
We finally obtained some modest support from the US DOD (DTRA) with the help of Mark Desrosiers from the National Institute of Standards and Technology. Somewhat to my horror, Mark began by trying first to convince the managers of the DTRA funding that they should also support using physical dosimeters, made by placing alanine into the standard buttons (pun intended!) of military uniforms, which I had assumed would be much more attractive to the military. However, the funding officers were soon convinced that military personnel would be tempted to manipulate their apparent exposure by changing their buttons, so they were much more intrigued by the concept of using the personnel's natural teeth as an accumulator of exposure, i.e., as a 'bio-dosimeter' that could not be artificially manipulated. While we achieved temporary success with the advocacy of these two funding officers, when they rotated to another assignment after a couple of years, the support from DTRA dried up.

We finally achieved a major breakthrough when the Centers for Medical Countermeasures for Radiation (CMCR) program of NIH was launched. Initially, we joined the team from the University of Rochester as one of their six projects in a CMCR that was funded for 5 years. Our budget was limited, but allowed us to start a wideranging EPR biodosimetry program with what, in retrospect, was a wildly ambitious set of goals laid out in the summary for our project:

The most urgent need is a field deployable instrument for rapid after-the-fact dosimetry. This will be our first priority. We initially will make the existing facility at Dartmouth fully operational for emergency dosimetry and will use these and further improvements as the basis for the rapid development of a transportable dosimeter suitable for field use. This will be tested at Rochester, where it also will be used for extending calibrations/validations, using the expertise and patient populations there. There also are a number of feasible ways to improve the sensitivity and field deployability of the method; these will be pursued as efficiently as possible without affecting the first priorities. These include the potential development of a hand-held version of the dosimeter, and several promising methods to enhance sensitivity. Such increases in sensitivity will further reduce the time needed to make the measurements and enable us to extend the method to a lower threshold, potentially to doses well below those that can cause acute clinical effects. There also are some plausible means to complement in vivo EPR dosimetry by making measurements in vitro, using hair, finger/toenails, or very small chips of teeth. These measurements could broaden the capacity for screening and also aid in resolution of asymmetric exposures.

The overall support for this project within the CMCR program at Rochester was certainly very significant (our support was about $\$ 400,000 /$ year for 5 years), but was spread out over a number of people and could not support new hires. Importantly, in the interim between getting the CMCR funded and getting the research up and going, we were able to communicate the potential for EPR biodosimetry fully and effectively to Dr. Mildred Donlon who was a program manager at DOD's DARPA. With her support, we were able to add substantially to our funding for in vivo tooth 
dosimetry and in vitro nail dosimetry (about $\$ 2,500,000$ direct costs spread out over 2 years).

Additionally, impressed by our success financially and the very supportive evaluations by the reviewers of our proposals, the administration of Dartmouth provided substantial additional funds to assure the stability of our core personnel. This support for the activities of engineers was crucial and unusual. Under the usual hypothesis-driven focus needed to obtain support from NIH, any funding of engineers needs to be rationalized as essential for solving the specific problems of the research. This policy seldom allowed full funding of key technical personnel and provided no path to their long-term stability. However, with the additional sources for support for our EPR Center, we were able to begin to assemble the required engineering expertise to develop the innovative and unique capabilities for in vivo EPR dosimetry and oximetry. Specifically, for biodosimetry, we could fully develop the instrumentation for the initial prototypes of instruments to measure radiation dose in vivo at L-Band in human subjects.

When the time came for competitive renewal of the CMCR Centers, we decided to go for our own CMCR at Dartmouth, devoted entirely to EPR dosimetry. There were several reasons to go solo. There were the usual stresses and complications in trying to work with a CMCR at Rochester located 350 miles away and with a very different orientation. In addition, in the renewal cycle of the CMCR Program at $\mathrm{NIH}$, the amount for each center was going to be decreased and Rochester had had six projects, five of which were local. They were therefore relieved to have us drop out.

Fortunately, our application for a focused EPR dosimetry CMCR was successful, with three projects funded on EPR dosimetry of in vivo teeth, in vitro nails (clippings), and in vivo nails. While the total per CMCR was indeed reduced, with no need to share with other projects at Dartmouth's CMCR, our laboratory enjoyed very substantial funding ( $\$ 3,300,000 /$ year total costs for 5 years).

About the same time, we were successful in getting our in vivo tooth dosimetry program funded by HHS' BARDA, for what turned out to be a total of more than $\$ 20,000,000$ over several years. These extraordinary levels of funding enabled us to retain and then substantially expand on the core engineering staff and, through the use of $\$ 300,000 /$ year of the funding for our CMCR that was designated for pilot projects outside of our laboratory, we were able to catalyze complementary instrumental and technique developments at other institutions, as well.

Eventually, we had more than 40 faculty and staff involved in the EPR program at Dartmouth in addition to graduate students doing research projects, Thayer School of Engineering students doing engineering projects, and Tuck School of Business students doing entrepreneurial projects. The EPR Center staff and faculty received partial or full support from grants and contracts (for which I was the Principal Investigator), which at their high point had a total budget of more than $\$ 10,000,000$ / year. While this funding was focused on EPR biodosimetry, the technology that was developed for in vivo dosimetry catalyzed the development of instrumental capabilities that greatly facilitated the development (and subsequent independent funding) of clinical EPR oximetry (detailed in a companion article ${ }^{572}$ ). 
The direction of our further developments of EPR biodosimetry was enormously influenced by the rationale behind the heavily funded federal initiative to develop medical countermeasures for potential exposures of large numbers of individuals to doses of radiation that had the potential to cause acute symptoms and possibly would be fatal. The large number of individuals who would be involved (many millions) required early and prompt identification of those at risk, so that the limited medical resources could be directed to those who potentially could benefit from them. It was recognized that biodosimetry was a key to identify rapidly whether an individual was at risk, so that high-risk individuals could be triaged for entrance into the medical system. An important contemporary consideration is the challenge of planning for biodosimetry in the event of a radiation event occurring simultaneously with another major disaster, such as a pandemic $^{557}$.

Through our own conceptualization of needs and from the mandates of the various federal entities involved in the development of medical countermeasures, we evolved our approaches using EPR-based biodosimetry to meet the needs associated with such a scenario. There are over 60 principal papers in biodosimetry from our laboratory $6,288,305,311,385,386,398-401,411-417,424,429,432$, $441,443,446-453,455,459-462,473,475-483,488,493-495,497,498,506-508,510,513,514,517,519$, $520,522-527,529,534,536,538,539,542,544$. As we focused on the practical aspects of using EPR dosimetry for rapid and effective triage, we developed techniques to assess the potential effectiveness of any biodosimetry methodology to be applied in the context of rapid triage under the circumstances of a large radiation incident $^{416,441,459,460,476,477,478,479,481,482, ~ 488, ~ 491, ~ 497, ~ 514, ~ 519, ~ 525, ~ 527, ~ 538, ~ 542 . ~ T h e s e ~}$ latter studies were especially led by Ann Flood.

The rationale for the high value of EPR dosimetry is based on the inherent characteristics of the radiation-induced changes that are detected with EPR. These characteristics derive from the fact that with EPR dosimetry, we measure a physical phenomenon directly in the individual in the part of the body in which we are interested, e.g., in teeth and nails. Because the changes that are induced by radiation occur essentially independently of any biological processing, we refer to these parameters as physically based biodosimeters. They are in contrast to biologically based biodosimeters whose measured parameters are very dependent on the past and current state of health and the physiological responses of cells and tissues to radiation damage.

Overall, because they are physically based, the favorable characteristics of in vivo EPR biodosimetry include

- Results are available immediately, i.e., the changes do not need to evolve for several hours or days before being detectable and do not continue to evolve.

- No need to remove the sample from the subject.

- Measurements can be made immediately after the event in the field and/or throughout the period of risk from acute medical effects from the exposure to radiation.

- EPR is capable of resolving the doses needed for effective triage, e.g., distinguishing above and below a meaningful threshold like 2 Gy. 
- EPR is capable of providing information on homogeneity of exposure (because it can be used to compare different sites on the body like teeth vs nails or hands vs feet or used in conjunction with biological measures to assess whether a single point assessment is identical to a systemic estimate).

- Unlike biologically based dosimetry techniques, EPR dosimetry will not be affected by other factors such as stress, concurrent wounds, and pre-existing conditions.

- Unlike biologically based biodosimetry, the timing and extent of changes do not vary with biological factors such as prior disease.

\subsubsection{Progress on In Vivo Tooth Dosimetry Since 2000}

Although we had previously presented information about the approach at several meetings ${ }^{288,305}$, our first publication reporting on the results from implementing in vivo tooth dosimetry was in $2000^{311}$. Even though this paper presented in vivo measurements made in rats, the spectrometer had been designed so as to be capable of making measurements in human subjects.

Finding suitable humans to measure was a challenge, since, unlike for animals, we could not experimentally expose humans to ionizing radiation. Our first published spectrum of a tooth measured in a human mouth was reported in $2005^{385}$; these measurements used an irradiated human tooth, which we inserted into the mouth of a volunteer who had had a gap in his teeth that could accommodate a fixture holding the irradiated tooth.

Partly based on this experience and the common use of molars in in vitro tooth dosimetry, for some time, we continued to develop the techniques aiming at measuring the radiation-induced EPR signal in molar teeth. Eventually we recognized that, if we were setting up our EPR tooth dosimetry device to be used for triage, we needed to change our approach and move from measuring molars to measuring upper central incisors.

There were several reasons for making this fundamental shift in our approach. In the scenario of a massive emergency event, measurements resulting in dose estimates would need to be made very quickly and simply by people who had no prior familiarity with EPR, and the instrumentation would need to be automated to produce the intended end result to the greatest extent feasible. Measurements in molars were physically awkward, requiring the operator to place the resonator in the back of the mouth to reach the molars, which takes skill and time, neither of which would be compatible with the aim of having a technique that could be used rapidly by minimally trained operators. We also conducted several timed mock-measurement sessions that were observed by emergency responders. These experts strongly recommended that we find a way to measure subjects while sitting up, to reduce the time needed to place a subject into the magnet ${ }^{451}$. We also recognized that, for many American adults, most or all of their molars have fillings, which of course would not be suitable for assessing dose. Also, the shape of the molars varied considerable among subjects, making it difficult to place the resonator consistently, especially if using the biting surface. 
The two front incisors, on the other hand, had several advantages. Their front surface varies less in its contours; indeed, the front surfaces are relatively flat, and therefore, it is easier to place the resonator rapidly and reliably on them. Incisors are less likely to have fillings (which can interfere with measurements) and are the earliest adult teeth to erupt (making these teeth potentially accessible to measure dose in children; deciduous teeth are not suitable, since they have a different type of enamel structure). Because of their location in the front of the mouth, they can be readily accessed by minimally trained personnel, and they easily accommodate measurements made with the subject sitting in front of a smaller (and lighter more easily transported) magnet. Indeed, as we later designed and implemented measurements using incisors, placement of the resonator could be automated ${ }^{525,567}$.

One of the potential problems of using incisors for dosimetry is the potential for confounding from exposure to the UV in sunlight, which can also induce EPR signals in teeth that are indistinguishable from those of ionizing radiation. The key issue is the levels of such exposures that could occur to the incisors over a lifetime. With our collaborators from Japan and Russia and carefully designed quantitative studies, we were able to show that, despite contrary assertions in some comments in the literature, UV exposure in teeth would not be a significant confounder ${ }^{494,566}$.

Eventually we refined the design to an almost fully automated device that could be utilized by personnel who had no prior experience with EPR. We have concluded that our device could provide a rapid (less than $5 \mathrm{~min}$ ) differentiation of individuals as to whether or not they may have received a medically acute radiation dose (i.e., greater than 2 Gy) that would indicate significant risk for having acute symptoms from ionizing radiation ${ }^{525}$.

\subsubsection{Current Status of In Vivo Tooth Dosimetry}

In the process of developing the current version of the tooth dosimeter, we examined a wide range of magnets, resonators, and dose calculating algorithms. Our grants and contracts enabled us to involve leading designers of permanent magnets in configurations that ranged from a one-sided magnet to an intraoral magnet. Eventually, we concluded that the best configuration is a dipole magnet with a gap sufficiently wide to have comfortable access for virtually any sized head. The resonator is placed on the front surface of one of the two upper central incisors. To avoid variations due to small differences in the shapes of the teeth and the placement of the resonator, the magnet is automatically rotated through a small set of angles. The calculation of the magnitude of the EPR signal is done by automated fitting of the EPR spectrum to the known shape of the radiation-induced EPR signal at L-Band frequency. A standard for field calibration and monitoring performance of the instrument is included in the sensitive volume of the resonator. The output includes a dose estimate and the uncertainty in that estimate.

There has been significant interest in our instrumentation and approaches, especially in Asia. The Radiation Health Research Institute in Korea, with responsibility for monitoring exposures from nuclear power plants and other inadvertent exposures to ionizing radiation, has obtained a clone of our tooth dosimeter and has been making measurements for several years. One of their goals was to establish baseline 
measurements for people living in the area or working at nuclear power plants for several years, but we are not aware of any publication of their data. Another group at Seoul University collaborated with us to develop their own version of in vivo tooth dosimetry instrument and has now published the first results using their system, characterizing the impact of geometry of teeth on EPR signals ${ }^{563}$. The most active group in dosimetry has involved our colleagues in Japan, led by Dr's Minoru Miyake, Hiroshi Hirata, and Ichiro Yamaguchi (all of whom have spent significant time in our Dartmouth lab). They have been using EPR systems derived from those at Dartmouth and made available through Clin-EPR, LLC ${ }^{3}$ as well using our resonators optimized in collaboration with Hiroshi ${ }^{484,495,555}$. Besides studying the potential confounding effects of UV exposure ${ }^{566}$, they have especially used EPR dosimetry to determine the radiation exposures in $\operatorname{man}^{523,536}$ and in beast ${ }^{554}$ that were associated with the 2011 nuclear power plant accident in Fukushima Prefecture.

While we enjoyed very high levels of financial support for our efforts to make in vivo tooth dosimetry into a device that would be adopted by civilian and military planners as one of the primary triage tools to be employed for managing a large event, to date, we have not succeeded in getting our device incorporated into the official response systems.

There are conceptual and practical barriers which we so far have not been able to overcome. Current plans for dosimetry favor the use of biologically based biodosimetry, despite the wide variation among individuals in their irradiation-induced responses due to their prior medical history and physiological variations and despite the difficulties of optimal timing of sample collection post-exposure and the complexity of carrying out analyses under emergency conditions and for a very large number of victims ${ }^{477,497}$. In contrast, in vivo EPR tooth dosimetry can meet the US technical specifications for point-of-service biodosimeters including providing a completed dose estimate in $5 \mathrm{~min}$, i.e., as timed from sampling the tooth (interrogating it with the resonator) to completing the EPR measurements and analysis for the triage decision-maker to use.

The primary concern of decision-makers, nonetheless, has focused on the fact that a single EPR dosimeter would have limited throughput (20 victims per hour per device) compared to high-throughput analysis devices available for biologically based dosimetry (which can process prepared samples by the thousands per hour per device). This of course ignores the times needed to collect the biological sample from the victim and prepare and transport it to the high-throughput device and then communicate the result back to the triage decision-maker who may have to track down the victim, because several hours or days will have elapsed. It also ignores the possibility of overcoming the throughput issue for EPR dosimetry by simultaneously using many devices in the field with the victim and triage decision-maker both present ${ }^{497}$. However, we will keep working to try to convince them of the advantages of EPR tooth dosimetry!

\footnotetext{
${ }^{3}$ Clin-EPR, LLC of Lyme, NH was founded by Hal \& Ann to enable the dissemination of in vivo EPR technology for investigational use that had been developed in our academic programs.
} 


\subsection{Measuring Fingernails and Toenails for Triage}

Our current emphasis is on the use of in vivo nail dosimetry which, in addition to sharing some of the advantages of in vivo tooth dosimetry, has the very significant additional advantage of providing dose at multiple points (i.e., using four limbs, with up to five digits per limb). This unique advantage of in vivo nails can provide crucial information for medical decision-making, which is whether the exposure was uniform or heterogeneous. If the dose was heterogeneous, e.g., the dose to the feet differed significantly from the hands, the medical consequences would likely be determined by the lowest dose received to part of the bone marrow. This capability would be especially valuable in determining whether to use radiation countermeasures and, if so, which ones to use ${ }^{558}$.

Because the principal cause of death from ionizing radiation at the doses of interest in such an event is failure of the bone marrow, knowing whether some parts of the body were spared high dose would be important for medical decision-making. Because the bone marrow is widely distributed, the critical dose for medical management is the lowest dose to a bone marrow producing region. That is because any significant functioning bone marrow can serve as an auto-transplant of bone marrow to damaged regions elsewhere.

We and others first tried to use nail clippings for dose estimates. These appeared to have many advantages, including the ability of the victim to provide their own samples. We turn next to our experience with nail clippings.

\subsubsection{In Vitro Clippings of Fingernails and Toenails}

Our laboratory at WRAIR appears to have been the first group to suggest the use of the radiation-induced EPR signal in nails as a biodosimeter and made measurements of nails in $1968^{6}$. As noted above, there are several obvious advantages of using clippings, including self-sampling, easy analysis using higher frequency EPR spectrometers and, because each hand and foot could be sampled, clipped nails could also be used to determine whether exposure was homogenous.

Subsequently, a number of excellent investigators have worked diligently independently of and also in collaboration with us ${ }^{412,413,432,448,452,462,493,508,522}$ to try to move EPR measurements of nail clippings from its possible advantages to being a practical tool to use for triage in a large-scale radiation disaster.

The principal complication in accomplishing this transition centers around the problem that the clipping process introduces mechanically induced EPR signals (MIS) in nails, i.e., when fingernails are cut, the cutting process generates a very strong and complex set of EPR signals that fully overlap the radiation-induced EPR signals (RIS) [7]. In addition, there are further qualitative and quantitative changes in the EPR signals that vary with the environment which the nails experience such as moisture and temperature. The potential for contaminants such as exposure to nail polish or grease added further complications. Eventually, we concluded that this initially attractive approach for dose estimation cannot be implemented in a dependable 
and useful way for large events, although it does remain useful for very small-scale events.

\subsubsection{In Vivo Nails on Fingers and Toes}

Because of the problems that we encountered in using nail clippings for triage as discussed above, we decided to carry out a dedicated and systematic effort to develop in vivo EPR nail dosimetry. Therefore, when we received our independent CMCR for EPR dosimetry at Dartmouth, one of our three major projects was in vivo nail dosimetry.

We recognized that in vivo nails, i.e., measuring the nails while still attached to the subject's nailbed, would be very demanding technologically. While the nails themselves are not very lossy, they are located in close proximity to the tissues of the finger (or toes) that cause non-resonant losses and degrade resonator efficiency.

For in vivo EPR tooth dosimetry, we were able to minimize non-resonant absorption (loss) of microwaves in tissues in the mouth by making the measurements at a lower frequency (at L-band or 1.2 GHz). This strategy worked with teeth because the EPR signal (RIS) in irradiated teeth was sufficiently intense to be measurable, in spite of the lower sensitivity that occurs with lower frequency.

In the case of nails, however, this was not an option because of the lower intensity of the RIS in nails. To overcome this problem and take advantage of the fact that the intensity of EPR signals increases linearly with frequency, we decided to use the readily available and well-known X-Band technology for our approach.

The remaining problem to solve was to figure out a way to at least diminish the non-resonant absorption of X-Band in the surrounding lossy tissues. The solution required developing innovative new EPR resonators that would limit the volume that is a 'sampled' to be (mostly) within the thickness of the nail plate, i.e., avoiding the underlying lossy tissue of the finger.

Without knowing technically how this could be achieved, I challenged Jim Hyde and his colleagues (especially Jason Sidabras) at the Medical College of Wisconsin (MCW) to develop the required technology. In parallel, Oleg Grinberg, an outstanding EPR physicist in our group, also took on this formidable task. Eventually, these brilliant colleagues developed two complementary approaches to allow us to make $\mathrm{X}$-Band measurements in the nail plates of fingers and toes. Prior to advancing these techniques and following the lead of Jason, we first developed expertise with finiteelement analysis to facilitate the development of the different resonant structures 450, 505, 524, 538, 550

Oleg's approach to solve this particular problem, working closely with Jason, was to adapt an existing technique, the aperture resonator ${ }^{524,550}$. He used resonators with either a hole or slot cut into the far wall, through which the magnetic component of the microwave would leak and against which the subject could place his/her digit with the nail adjacent to the opening.

Several innovative approaches for this type of resonator were advanced including the use of a non-resonant dielectric slab aperture resonator (DAR). The dielectric slab provides an increased magnetic field at the aperture and sample, while 
minimizing the sensitivity of the aperture resonance to the load conditions. We also introduced a DAR semi-spherical (SS) $\mathrm{TE}_{011}$ geometry. The SS-TE 011 geometry is attractive due to having twice the incident magnetic field at the aperture for a fixed input power ${ }^{505}$.

This approach was shown to have considerable promise and should be further investigated, but as a matter of expediency to meet the requirements of our external funders who wanted more prompt results, we have focused the approach developed by the team at MCW. They conceptualized and then developed practical versions of a unique surface resonator array (SRA) for use in EPR; (SRAs had previously been used for NMR, in a different context $)^{505}$. The attractiveness of this approach lies in the ability to design, construct, and test the SRAs for measurements at X-Band in which the sensitive region is sufficiently confined to the nail plate, so that radiation levels of 1 Gy or lower can be measured.

In vivo nail EPR dosimetry using SRAs has become our leading approach in using EPR to carry out triage after a major incident. The reason that this approach is potentially so valuable is that it can determine whether or not someone is at risk of acute clinical effects of ionizing radiation, by both estimating the magnitude of the dose and by determining whether the exposure was homogeneous. Currently, there are no other biodosimetry methods that can give both types of information as well as can in vivo EPR dosimetry of nails.

Additional positive features of in vivo EPR nail dosimetry include the ability to increase sensitivity (e.g., by independently measuring more than one nail on a hand or foot and combining these assessments) and to automate the device and speed up spectra acquisition (e.g., by making simultaneous measurements on all four limbs). And, of course, EPR nail dosimetry shares several features of tooth dosimetry such as having estimates that would be unaffected by simultaneous other injuries, having diagnostic changes that occur instantly after the irradiation, and minimizing variability in individual responses to the same exposure. Although it remains to be shown definitively, evidence to date suggests that the presence of nail polish (if removed) or infections in the nails will not have a major impact on the reliability of the method. However, of course, at this time, the full development of automated nail dosimetry has not occurred, so there is much work left to do to establish that this approach will indeed be widely adopted.

\section{Conclusions and the Current and Future State of EPR Dosimetry}

From the points of view of science, logistics, and practicality, EPR dosimetry has many positive characteristics that cannot readily be achieved by other methods. For this reason, it is reasonable to remain optimistic that eventually this will be widely recognized by the appropriate decision-makers, both military and civilian.

While not fully prototyped, the most useful and therefore the EPR technique most appropriate to adopt first is in vivo nail dosimetry. Its usefulness lies in its irreplaceable role in determining the medical implications of a radiation exposure ( 250-600 cGy) where treatment could significantly impact survival. EPR nail dosimetry could inform the triage and clinical decision-makers about the magnitude 
of the exposure and whether/where there are areas of bone marrow likely to provide life-saving auto transplantation of bone marrow to the rest of the body. Then, informed decisions could be made regarding the need for any treatment and the type of treatment that is indicated, including mitigators. And, where medical resources are limited, it can provide information needed to further prioritize treatment for the individual.

Tooth dosimetry is likely also to be valuable for determining the likely level of dose if in vivo nail dosimetry is not available. Further progress can be made, because the basic technology has been developed and current prototypes need only modest modifications. Equally important, the potential of EPR biodosimetry is now widely appreciated and built into the fabric of discussions of biodosimetry, even if particular applications or approaches may be over- or undervalued.

Acknowledgements These studies and instrumental developments are the product of many people's efforts as well as funding from many sources over the past 50 or so years. While I have named several special contributors in the context of this history, I probably have not been thorough, so the reader should note the many coauthors of my papers for a fuller picture of the key players. Most importantly, I would like to acknowledge the contributions of my most important partner and colleague (and wife): Ann Barry Flood. She has provided her always helpful criticism and editorial contributions as well as her very substantial roles in the academic content covered in this history.

\section{Declarations}

Conflict of interests Harold Swartz and Ann Flood are co-founders of Clin-EPR, LLC which makes in vivo EPR instruments for preclinical and clinical investigational use only.

\section{References}

1. A.B. Flood, S.G. Swarts, M.C. Krishna, B. Gallez, Appl. Mag. Res. In Press, (2022)

2. W. Gordy, H. Shields, Radiat. Res. 9, 611-625 (1958)

3. R.O. Becker, Nature 199, 1304-1305 (1963)

4. T. Cole, A.H. Silver, Nature 200, 700-701 (1963)

5. J. Duchesne, J. Depireux, J.M. Van Der Kaa, Geochim. Cosmochim. Acta 23, 209-218 (1961)

6. C. Yamanaka, M. Ikeya, H. Hara, Appl. Radiat. Isot. 44(1-2), 77-80 (1993). https://doi.org/10. 1016/0969-8043(93)90199-k (PMID: 8386054)

7. N. Tkatchenko, A. Romanyukha, R. Reyes, S.G. Swarts, D. Gourier, F. Trompier, Appl. Magn. Rad. 51(1), 1 (2022)

Publisher's Note Springer Nature remains neutral with regard to jurisdictional claims in published maps and institutional affiliations. 